\title{
The Research in Reasonable Development Mode of Volcanic Fractured Heavy Oil Reservoir: A Case Study of Zao 35 Fault Block
}

\author{
Ji Ling \\ (The Third Oil Production Company of Dagang Oilfield, Cang County 061035, China)
}

\begin{abstract}
China, and become a hot area of exploration and development in recent years. Due to the dual or even multiple porous media of volcanic reservoirs and complex reservoir spaces and construct types, it is extremely difficult to development this type of reservoirs. There is not a systematic and mature development mode in the world at present. Reports on the exploitation of volcanic reservoirs are few and reports on the volcanic fractured heavy oil reservoir exploitation are even fewer. Zao 35 fault block of Zaoyuan oilfield is a heavy oil reservoir with complex reservoir spaces, construct types, bottom-water, no edge-water and natural energy exploitation in initial stage. Due to insufficient formation energy, the production rapid declines. Some different field tests such as water drive and microbe drive were carry out later. In this paper, taking Zao 35 as an example, the reasonable volcanic fractured heavy oil reservoir development modes are discussed basing on different field tests analysis combined with numerical reservoir simulation study.
\end{abstract}

Keywords: volcanic rock, fracture, heavy oil reservoir, development mode

In the past half century, although the volcanic reservoirs have been found continuously, but the total and the scale are still small ${ }^{[1]}$. There is still a large amount of volcanic reservoirs in eastern and western China (Daqing, Liaohe, Dagang, Xinjiang, Sichuan, Shengli). As these reservoirs have been discovered and developed, volcanic reservoirs have increasingly become the focus of exploration and development. At present, there are not many examples of volcanic reservoirs ${ }^{[2]}$, and there are fewer reports on the exploitation of volcanic fractured heavy oil reservoirs. Zao 35 Block of Dagang Zaoyuan Oilfield is typical volcanic fractured heavy oil reservoir, with dual media of fracture - hole. It has its particularity and its development and law are different from conventional heavy reservoir. Therefore, the study of this block can provide some valuable experience for similar reservoirs development.

\section{Oilfield Situation}

Zao 35 block is located in drop plate of Li Tianmu Fault of Zilaitun Structure which is in the north of Fenghuadian Structure in Huanghua Depression. The geological structure is a monocline plunging from north west to south east. Its northwest part is blocked by Li Tianmu Fault with north east extension. It is a volcanic pore fractured heavy oil reservoir which belongs to a typical type of complex special reservoir (Figure 1). Sha-3 member is the oil bearing layer. The reservoir depth is $1470 \sim 1695 \mathrm{~m}$, the oil-bearing area is $2.48 \mathrm{~km}^{2}$ and the geological reserves are about $713 \times 10^{4} \mathrm{t}$. It consists of explosive facies, overflow facies and breccia facies, with lithology of basalt, tuff and breccia volcano. Fracture system develops with the main fracture of NE and NW. The reservoir space is mainly pore and fracture, matrix porosity porosity is $21.6 \% \sim 34.4 \%$, fracture-cavity porosity is $1.18 \% \sim 4.4 \%$.

The characteristics of the crude oil in the Zao 35 block are high density, high viscosity, high asphaltene content, high initial boiling point and low wax content. The average density is $0.9732 \times 10^{3} \mathrm{~kg} / \mathrm{m}^{3}$. Under the reservoir conditions, the viscosity is $1449.59 \mathrm{mPa}$.S, the wax content is $5.5 \%$, the average gum asphaltene content is $35.94 \%$, and the average initial distillation point is 188.1 centigrade, and the average wax content is as high as $5.5 \%$ (Table 1$)$.

Based on the analysis of high pressure physical samples, the formation oil of the volcanic reservoir in Zao 35 block is characterized by low saturation pressure, medium compressibility, low volume coefficient, low gas oil ratio and high density. The original saturation pressure of formation oil is $4.41 \mathrm{MPa}$, the compression coefficient is $17.05 \times 10^{-4} \mathrm{MPa}$, the volume coefficient is about, and the gas oil ratio is $9 \mathrm{~m}^{3} / \mathrm{m}^{3}$, the density of formation oil is about $0.896 \times 10^{3} \mathrm{~kg} / \mathrm{m}^{3}$.

In Zao 35 block, average effective thickness is $40 \mathrm{~m}$, porosity is $20 \%$, permeability is $375 \times 10^{-3} \mu \mathrm{m}^{2}$. The average depth of reservoir is $1561.4 \mathrm{~m}$, the average temperature is 60 centigrade, the average original formation pressure is $17.86 \mathrm{MPa}$. 
The Research In Reasonable Development Mode Of Volcanic Fractured Heavy Oil Reservoir...

Table 1 Physical property statistics of crude oil in Zao 35 block

\begin{tabular}{|c|c|c|c|c|c|c|}
\hline \multirow[t]{2}{*}{ Well } & \multirow{2}{*}{$\begin{array}{l}\text { Well Section } \\
(\mathrm{m})\end{array}$} & \multicolumn{5}{|l|}{ Oil Property } \\
\hline & & $\begin{array}{l}\text { Density } \mathrm{g} / \mathrm{cm}^{3} \\
\left(20^{\circ} \mathrm{C}\right)\end{array}$ & $\begin{array}{l}\text { Viscosity MPa.s } \\
\left(80^{\circ} \mathrm{C}\right)\end{array}$ & $\begin{array}{l}\text { Freezing Point } \\
\left({ }^{\circ} \mathrm{C}\right)\end{array}$ & $\begin{array}{l}\text { Wax Content } \\
(\%)\end{array}$ & $\begin{array}{l}\text { gum asphaltic } \\
(\%)\end{array}$ \\
\hline Jun21-19 & $1718.9-1755.5$ & 0.9669 & 921.0 & 13 & 3.53 & 45.84 \\
\hline Jun 21-23 & $1574.2-1642.4$ & 0.9651 & 1152.7 & 23 & 8.74 & 40.60 \\
\hline Jun 23-19 & $1627.0-1639.0$ & 0.9923 & 839.0 & 10 & 2.62 & 48.57 \\
\hline Jun 23-21 & $1542.5-1644.6$ & 0.9663 & 820.5 & 12 & 1.89 & 46.28 \\
\hline Jun 23-25 & $1549.8-1628.0$ & 0.9688 & 1167.0 & 16 & 8.45 & 42.50 \\
\hline Jun 25-27 & $1528.4-1634.3$ & 0.9710 & 3629.7 & 22 & 1.47 & 46.40 \\
\hline Zao35 & $1507.1-1541.0$ & 0.9480 & 692.9 & 13 & 3.64 & 45.65 \\
\hline Zao 78 & $1495.0-1531.2$ & 0.9664 & 692.2 & 3 & 4.88 & 43.89 \\
\hline Zao 79 & $1548.0-1571.0$ & 0.9647 & 1862.8 & 15 & 1 & 1 \\
\hline Zi6-12 & $1583.0-1622.0$ & 0.9904 & 1 & 28 & 2.35 & 48.48 \\
\hline Zi6-14 & 1588.4-1623.0 & 0.9610 & 870.9 & 24 & 3.94 & 45.91 \\
\hline Jun 21-27 & $1578.7-1612.9$ & 0.9813 & 3296.8 & 19 & 6.25 & 44.58 \\
\hline Jun 25-23 & $1468.1-1557.0$ & 1.0067 & 1 & 55 & 1 & 1 \\
\hline
\end{tabular}

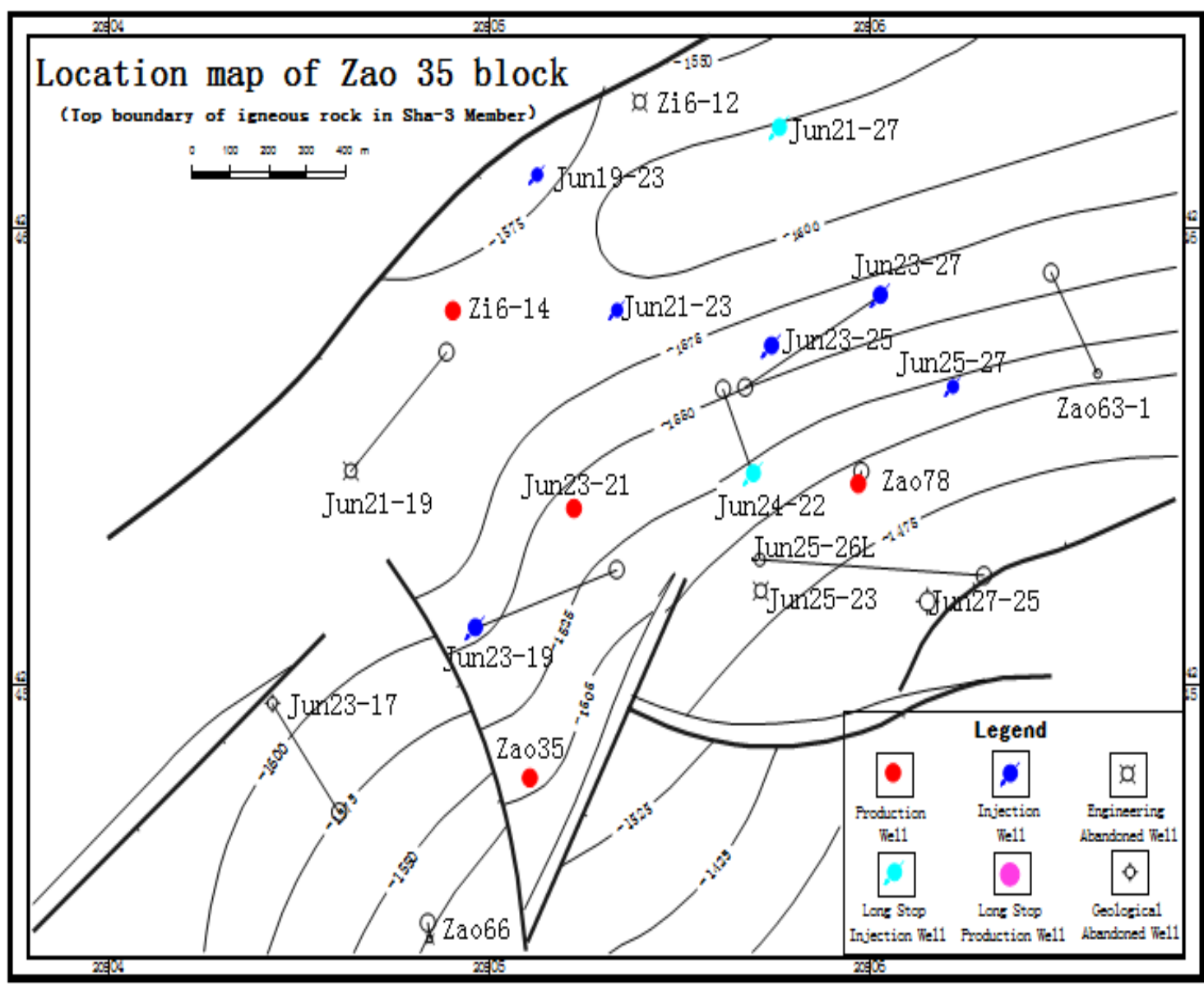

Figure 1 Location map of Zao 35 block

\section{Seepage Mode}

The seepage channels of volcano rock are complex with both pore throat and factures, even bead shaped stomatal. There are many types of relationship between reservoir space and seepage channel. On the basis of the relationship between the reservoir space and the seepage channel, two main reservoir seepage mode can be concluded ${ }^{[3]}$ :

(1) fracture-pore reservoir seepage mode (Figure 2A). Matrix intergranular pores are the main reservoir space, micro fractures are the main seepage channel, tuff of eruptive facies and breccia belong to this kind of seepage mode.

(2) large pore and joint-small pore and joint composite reservoir seepage mode (Figure $2 \mathrm{~B}$ ). Small stomatal and small joint constitute the matrix system, large pore and joint constitute the fracture system, forming large pore and joint-small pore and joint composite double medium reservoir seepage mode, the overflow facice of stomatal basalts belongs to this kind of reservoir seepage mode. 

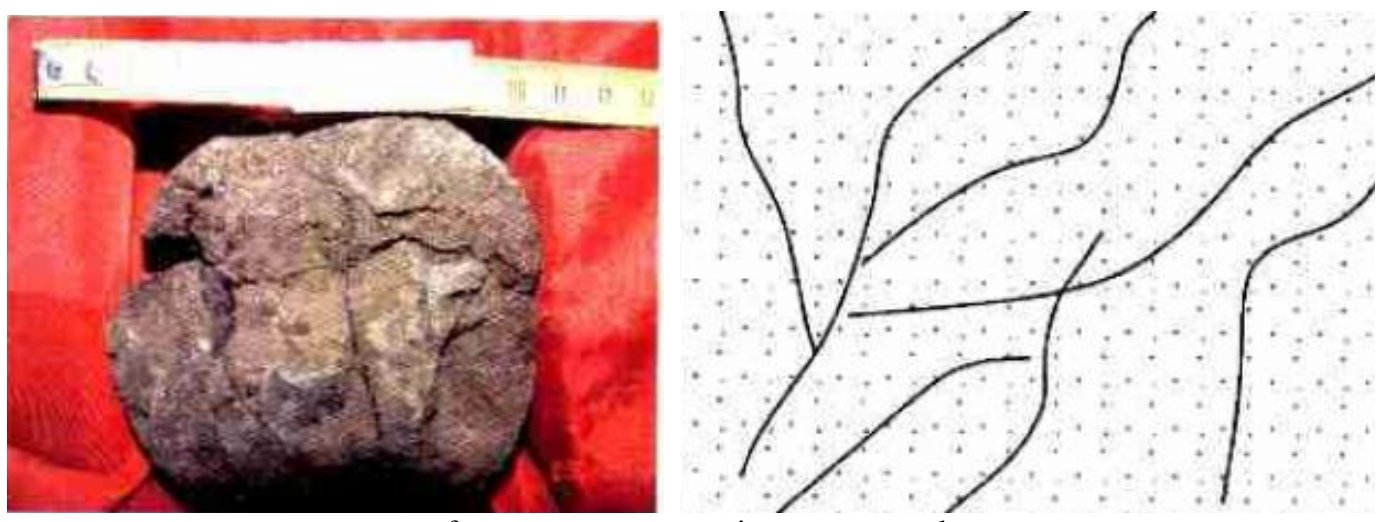

a fracture-pore reservoir seepage mode
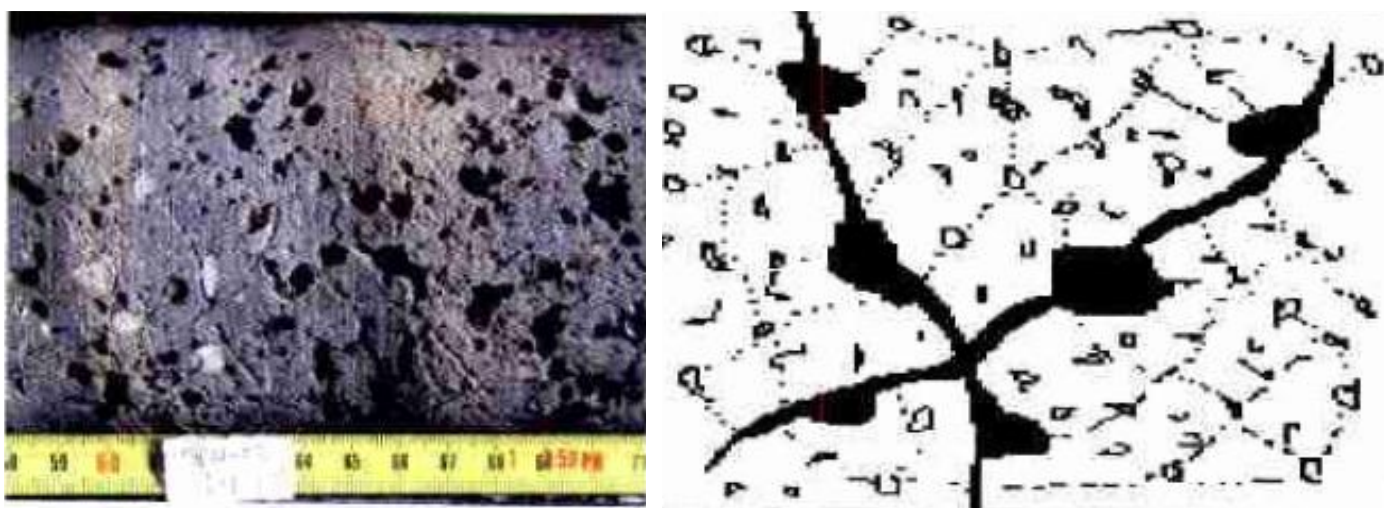

b large pore and joint-small pore and joint composite reservoir seepage mode

Figure 2 Reservoir Seepage Mode

\subsection{Depletion Development}

III. Practice Effect of Different Development Methods

The depletion development mainly depends on the fracture closure, the reduction of the matrix pore size and the expansion of oil to displace oil. Zao 35 block is in depletion development stage from January 1996 to May 1997, 12 wells are in production including the opening of 9 . In the early stage, the maximum output of each well reachs $81.7 \mathrm{t} / \mathrm{d}$, and the cumulative oil production was $19.83 \times 10^{4} \mathrm{t}$.

Due to the rapid decline of formation pressure caused by depletion development (the ground pressure decreases from $17.86 \mathrm{MPa}$ to $6.5 \mathrm{MPa}$ in 1 and a half years), the fracture closure seriously affectes the production capacity of wells. At the same time, due to poor mobility of heavy oil, the recovery rate of the depletion development is only $3.5 \%$ (Figure 3 ).

\subsection{Waterflood Development}

In the past 15 years (From June 1997 to present) of waterflood development, the cumulative oil production is about $25.7 \times 10^{4} \mathrm{t}$, and the recovery rate is up to $6.2 \%$, and the comprehensive water cut rate is as high as $90 \%$ in Zao 35 block. Through the relationship between water cut rate and recovery percent (Figure 3), the characteristic of high oil water viscosity ratio reflects the viscous fingering phenomenon of fractured heavy oil reservoir.

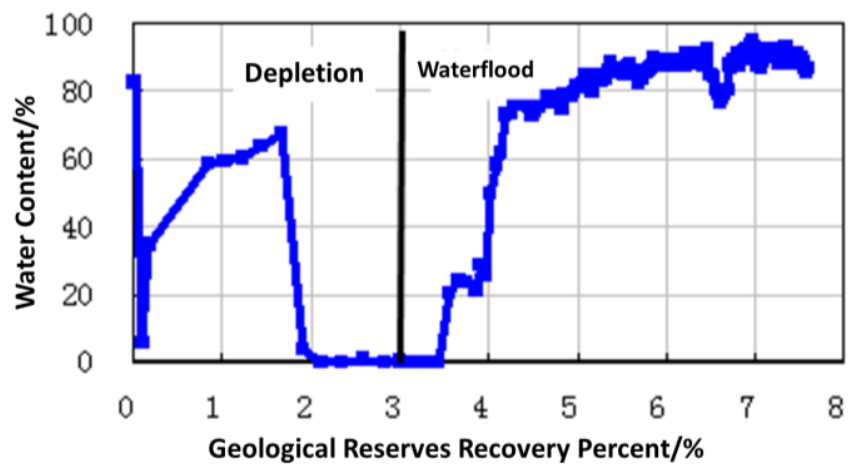

Figure 3 The relationship between water cut rate and recovery percent in Zao 35 block 
In the course of water flooding, fracture system (large joint and hole) mainly depends on the displacement effect of oil discharge, but the matrix system (small large joint and hole) mainly depends on the capillary force of oil discharge. But for the fractured heavy oil reservoir, due to the serious water channeling, the injected water can only drive some of the crude oil in the fractures, while the crude oil in the matrix is difficult to rely on imbibition and injection water to be drived out. Because of the Zao 35 block is adouble medium heavy oil reservoir with fractures and pore, its poor physical property of crude oil, high oil-water viscosity ratio, leading to the strong non piston of injected water flooding front, serious viscous fingering, coupled with strong reservoir heterogeneity, injected water channeling along the high permeability layer especially along the fractures. Then the channel is formed between oil well and water well, water injection is ineffective circulation, serious water out happens in oil wells.

In the Zao 35 block, after water flooding in the volcanic fractured heavy oil reservoir, no effect of increasing production produces, and the production decline rate is still large, but the trend of the sharp decline of the formation pressure has been controlled and begun to slowly recover. The injected water flows along the fracture, the oil wells are suddenly flooded, the water content of the block rises rapidly, and the high water cut development stage comes only 15 months after the injection. The recovery rate of water flooding is low, and the reservoir potential is not fully exploited. The application of the water flooding method in the exploitation of volcanic fractured heavy oil reservoir is not effective.

\subsection{Microbial Flooding}

The Zao 35 block is a volcanic reservoir, there is no formation water at the end of the elastic production and the formation permeability is strong. The experiment of oil displacement in the room also proves that no matter water flooding, polymer flooding or alkali + active oil displacement, the displacement liquid quickly eject. According to the reservoir conditions, in 2003, according to the characteristics of the heavy oil reservoir and the characteristics of microbial oil displacement in the Zao 35 block, the microbial test is carried out, setting up the microbial flooding test ${ }^{[4]}$.

After the microbial test, on the one hand, the oil well output fluid do not reduce because of inadequate supply of oil in the formation, $200 \mathrm{~m}^{3}$ large displacement pump is still used in production; on the other hand, under the condition of the high rate of production, liquid supply can meet, and the yield increase with no formation water. The results shows that the growth, migration, production of various substances of microbial in the reservoir increase the flow coefficient of crude oil, increase the pore pressure of the rock, thereby enhance oil recovery.

\section{Research on Reasonable Development Mode}

The way of reasonable development of volcanic fractured heavy oil reservoir need to be addressed several issues: one is to solve the problem of fracture water channeling, water flooded; one is to reduce the viscosity and increase its mobility; one is to solve the problem of efficient exploitation of matrix crude oil; one is to solve the problems of deep buried heavy oil reservoir ${ }^{[5 \sim 6]}$. Because of the small amount of crude oil in the fracture, it is easy to exploit for the volcanic fractured double medium heavy oil reservoir. Therefore, the effective development of crude oil in the matrix is an urgent problem to be solved.

\subsection{Establishment of mechanism model}

According to the actual fluid parameters of Zao 35 block, a typical mechanism model is established. The grid system using three-dimensional cartesian grid is divided into 40 grids, the direction of $\mathrm{X}$ is $5 \mathrm{~m}$, the direction of $\mathrm{Y}$ is divided into 40 grids, the step length is $5 \mathrm{~m}$, and the $\mathrm{Z}$ direction is divided into 20 grids, the step length is $2 \mathrm{~m}$. The total area of the simulated area is $200 \mathrm{~m} \times 200 \mathrm{~m}=40000 \mathrm{~m}^{2}$, and the simulated grid system is about $40 \times 40 \times 20=32000$. In the model, 4 vertical wells are used as production wells, and 1 wells are used as injection wells (Figure 4).

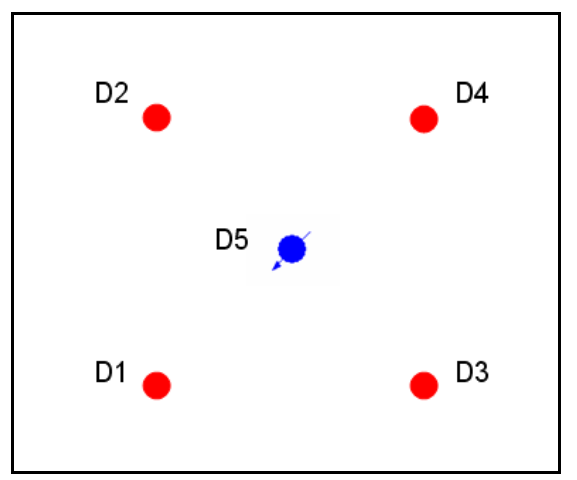

Figure 4 Well map of mechanism model 
The Research In Reasonable Development Mode Of Volcanic Fractured Heavy Oil Reservoir...

\subsection{Numerical simulation design}

In this paper, on the basis of the understanding of the field practice, 4 numerical simulation schemes are designed to study the effects of the four development modes of depletion development, water flooding development, steam huff and puff and steam flooding (Table 2).

Table 2 Numerical simulation design

\begin{tabular}{|l|l|l|l|l|}
\hline $\begin{array}{l}\text { Project } \\
\text { name }\end{array}$ & $\begin{array}{l}\text { Development } \\
\text { mode }\end{array}$ & $\begin{array}{l}\text { Maximum oil } \\
\text { yield } \mathbf{~ m}^{\mathbf{3} / \mathbf{d}}\end{array}$ & $\begin{array}{l}\text { Maximum liquid } \\
\text { yield } \mathbf{~ m}^{\mathbf{3} / \mathbf{d}}\end{array}$ & Production mode \\
\hline Project 1 & $\begin{array}{l}\text { depletion } \\
\text { development }\end{array}$ & 14 & 41 & $\begin{array}{l}4 \text { production wells in depletion development for 10 } \\
\text { years }\end{array}$ \\
\hline Project 2 & $\begin{array}{l}\text { water flooding } \\
\text { development }\end{array}$ & 14 & 41 & $\begin{array}{l}\text { After 4 production wells in depletion development for } \\
\text { 2 years, injection water (injection production ratio 1:1) }\end{array}$ \\
\hline Project 3 & $\begin{array}{l}\text { steam huff and } \\
\text { puff }\end{array}$ & 20 & 60 & $\begin{array}{l}\text { 4 production wells in steam huff and puff for 10 years, } \\
\text { 1 year is a cycle }\end{array}$ \\
\hline Project 4 & steam flooding & 20 & 60 & $\begin{array}{l}\text { After 4 production wells in depletion development for } \\
\text { 2 years, injection steam (injection production ratio 1:1) }\end{array}$ \\
\hline
\end{tabular}

\subsection{Numerical simulation results}

According to the results of numerical simulation, it can be seen that there is little difference between the recovery rate of water flooding and the depletion development, while the steam stimulation and steam flooding can greatly improve the recovery rate(Figure 5). For the fractured heavy oil reservoir, although the moisture content of depletion development is low, but the formation energy decreases quickly, the recovery rate is only $9.2 \%$; water flooding can supplement the formation energy, but because of the existence of fractures, water channeling is serious, water rise quickly, the recovery rate is only $10.4 \%$, compared to the depletion development, the recovery rate only increases by $1.2 \%$; steam stimulation can not only timely supplement energy, but also can inhibit gas channeling and rush to a certain extent, the recovery rate is $15.5 \%$, compared with the depletion development, recovery rate can be increased by $6.3 \%$. Steam flooding on the one hand can replenish the formation energy, taking the formation pressure stable at a high level, on the other hand can use the reserves of the matrix part, but also exist the phenomenon of steam channeling along the fractures, but the recovery rate is highest, $26.1 \%$.

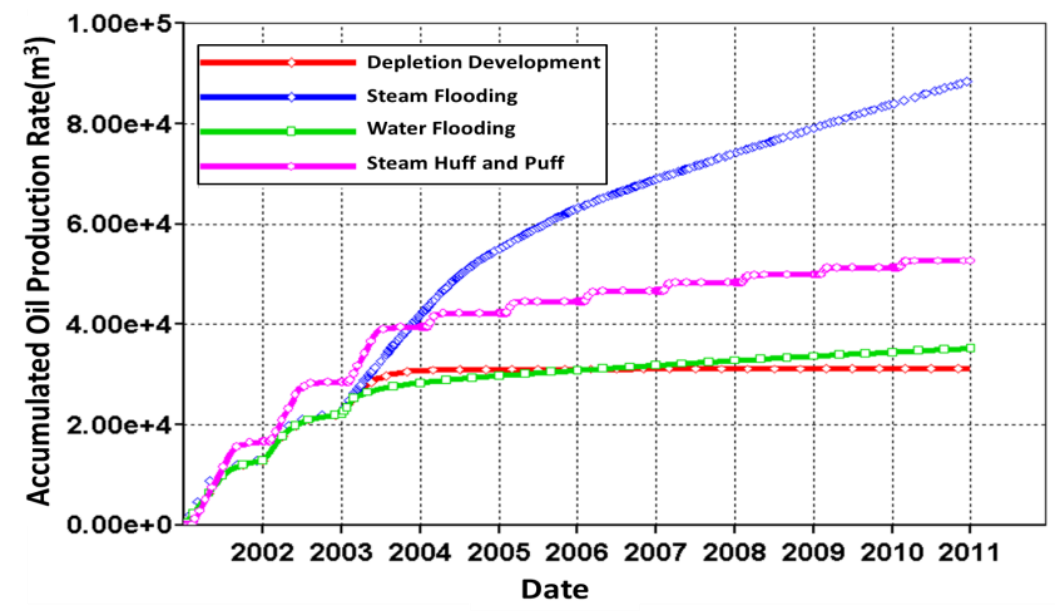

Figure 5 Comparison of cumulative oil production of different development mode

Table 3 Numerical simulation design

\begin{tabular}{|l|l|l|}
\hline Project name & Development mode & Ultimate recovery rate \% \\
\hline Project 1 & depletion development & 9.2 \\
\hline Project 2 & water flooding development & 10.4 \\
\hline Project 3 & steam huff and puff & 15.5 \\
\hline Project 4 & steam flooding & 26.1 \\
\hline
\end{tabular}

In the process of depletion development, the matrix and fracture system are elastic and plastic deformation, the pore volume of the matrix is reduced, and the fracture is closed, displacing crude oil from fractures and matrix systems.

In the fractured heavy oil reservoir of the water flooding process, the injected water can only drive part of the fractures in the matrix, but cannot drive the crude oil, and because the high viscosity of the crude oil cannot occur imbibition, crude oil of matrix cannot be produced in water flooding. Therefore, in the early development of volcanic reservoirs, generally water flooding is not used. 
The steam flooding can greatly improve the recovery rate of heavy oil reservoir, but the technology is still immature in deep heavy oil reservoir, which is still in the pilot stage, and has not been applied in large-scale industrialization. Especially for deep fractured heavy oil reservoir, the technology of steam flooding is more difficult. Because of the fractures and serious steam channeling, mining effect and economic benefit are not good.

Therefore, the steam huff and puff method is an effective way to exploit the fractured heavy oil reservoir. Its main advantages are: (1) viscosity and thermal expansion can greatly reduce the viscosity of crude oil heating heat injection process, greatly enhance the flowability of the crude oil; (2) during the process of steam stimulation, pressure disturbance can enhance the imbibition and flooding effect of matrix system; (3) for fractured reservoir, steam stimulation can reduce the fracture water channeling; (4) steam stimulation can also change the unfavorable factors of fracture for favorable factors, because the development of fracture and rock matrix is small, the steam flow in fractures and matrix contact area is bigger, the thermal efficiency of steam heating of the substrate is higher, which is good for exploitation of crude oil in the matrix.

\section{Conclusion and Suggestion}

(1) The field practice of different development methods of Zao 35 block shows that the formation pressure of the volcanic fractured heavy oil reservoir decrease quickly, the mobility is poor, and the recovery ratio of the depletion development is low. In the later period, the formation energy is maintained by water injection, due to the serious water channeling in fractures, the development effect is poor and the recovery rate is low. The results of microbial oil displacement test shows that the microorganism can increase the flow coefficient of the oil and increase the pore pressure of the rock, which can increase recovery rate to some extent but its effective time is short.

(2) According to the results of numerical simulation, it is known that the recovery rate of water flooding is not much different from that of depletion development, while the steam stimulation and steam flooding can greatly improve the recovery efficiency. The steam flooding can greatly improve the recovery of heavy oil reservoir, but the technology is still immature in deep heavy oil reservoir, which is still in the pilot stage, and has not been applied in large-scale industrialization. Therefore, the steam huff and puff method is an effective way to exploit the fractured heavy oil reservoir.

(3) For the fractured heavy oil reservoir, the oil recovery mechanisms of depletion development, water flooding, steam stimulation and steam flooding are obviously different. From the field practice and numerical simulation study, the replacement method after the depletion development should choose steam huff instead of water flooding.

\section{Reference}

[1]. Pan Zhongxiang Pan Zhongxiang Anthology of petroleum geology [M] Beijing: Petroleum Industry Press, 1989.

[2]. He Yan, to Wu, Wu niansheng. Volcanic oil and gas reservoir research. [J] Petroleum geology and development of Daqing, 1999,18 (4): $6 \sim 14$

[3]. Yuan Shiyi, Ran Qiquan, Hu Yongle, et al. Effective development method of volcanic fractured heavy oil reservoir [J] Petroleum Journal, 2005,26 (4): 63 68.

[4]. Sun Jianping, Ran Qiquan, Shi Huandian, et al.The waterflooding characteristic and effect evaluation of volcanic fractured heavy oil reservoir in Zao 35 block in Dagang Oilfield [J] PGRE, 2005,12 (1): 59 62

[5]. Wang Yizhong, Yuan Shiyi, et al. Numerical simulation of oil recovery mechanism in volcanic fractured heavy oil reservoir of Zao35 block in Dagang Oilfield [J] petroleum exploration and development, 2004,31 (4): 105 107

[6]. Wu Youjia. Study on the injection dynamic characteristics of volcanic reservoir [J] Journal of Southwest Petroleum Institute, 2001,23 (2):14 18 